\title{
Squirrel Search Algorithm for Solving Optimal Reactive Power Dispatch Problem with FACTS Device
}

\section{Balasubbareddy, Divyanshi Dwivedi}

\begin{abstract}
In this paper, a novel algorithm which is being inspired by the natural foraging phenomenon of squirrel, called as Squirrel SSA for solving optimal reactive power dispatch (ORPD) problem of power system, in which FACTS device namely, UPFC is incorporated. Power Injection Modeling (PIM) and Current Injection Modeling (CIM) of UPFC are considered, both are compared for determining the best modeling technique of UPFC which can be incorporated in power system. The performance and possibility of the proposed algorithm are validated on IEEE 30-bus power system. Results obtained are compared with the other recent algorithms to show the superiority of SSA.
\end{abstract}

Keywords : Squirrel Search Algorithm (SSA), ORPD problem, UPFC, generation fuel cost, transmission line losses.

\section{INTRODUCTION}

In the recent years, power system's development, mainly transmission network has been increased because of higher requirement in industries and deregulation. Thus, new ways to maximize the power transfer and effectively using existing transmission network has become an important aspect which is to be considered with balancing the acceptable levels of the stability. One of the ways is to incorporate the latest emerging power electronics devices i.e., FACTS (flexible AC Transmission system) controllers including TCSC, SSSC and UPFC which can vary the system parameters effectively, the detailed explanation regarding these devices are referred from [1].

On the other hand, solution of OPRD problem lead to improvise the planning and operation in the power system. Usually, OPRD is considered as a complex, nonlinear problem that can minimize the general objectives including generation fuel cost and transmission line losses. This can be achieved by identifying optimal values of control parameters which includes generator's voltage, tap-changing transformers, and shunt capacitors. Whenever power system is operating, the changes in demand exhibit to vary the generation of reactive power and then load voltages also suffer fluctuations.

Revised Manuscript Received on January 30, 2020.

* Correspondence Author

M Balasubbareddy*, Dept. of EEE, Chaitanya Bharathi Institute of Technology, Hyderabad, India. Email: balasubbareddy79@gmail.com

Divyanshi Dwivedi, Dept. of EEE, Chaitanya Bharathi Institute of Technology, Hyderabad, India. Email: divyanshidw@gmail.com

(C) The Authors. Published by Blue Eyes Intelligence Engineering and Sciences Publication (BEIESP). This is an open access article under the CC-BY-NC-ND license http://creativecommons.org/licenses/by-nc-nd/4.0/
The voltages may be adjusted by suitable reactive power controlling devices. For solving the OPRD problems, a number of classical and metaheuristics optimization techniques have been developed by researchers in past decades. Classical techniques include gradient methods, linear programming, nonlinear programming, quadratic programming, interior point and Newton formulation [2-5], but these techniques cannot be used to solve problem of large-scale power systems and sometimes it led the solution to be stuck in local minima. On the other hand, the metaheuristic optimization techniques such as genetic algorithm [6], GSA [7], simulated annealing (SA) [8], grey wolf optimizer [9], PSO [10], ABC algorithm [11] and many more algorithms had been developed which are considered as intelligent algorithms which help to overcome the problem faced in classical approaches.

Similarly in this paper, a novel squirrel search algorithm [12] is used for solving OPRD problems with incorporation of FACTS device i.e. UPFC. This paper deals to identify the most suitable way of modeling UPFC among PIM and CIM [13]-[14] of UPFC and furthermore optimal values for considered objectives is compared with existing algorithms for IEEE 30-bus standard test system.

\section{STEADY STATE MODEL OF UPFC}

\section{A. Power Injection Modeling of UPFC}

PI model of UPFC is referred from [13] is shown in Fig. 1. This modeling helps to understand the effect of the UPFC on the system in the steady state condition. Furthermore, it can be incorporated in the power flow model easily. The modeling of UPFC is expressed as

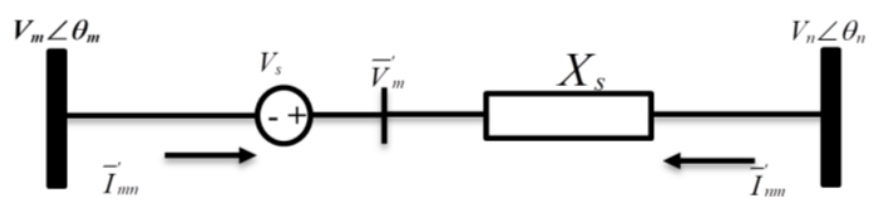

Fig. 1. Representation of VSC of UPFC where, $\bar{V}_{m}^{\prime}=V_{s}+V_{m}$ and $V_{s}=r V_{m} e^{j \gamma}$, directs the limits for the operation of UPFC which are: $0 \leq r \leq r_{\max }$ and $0 \leq \gamma \leq 2 \pi$. Final elements of equivalent power injections are:

$$
\begin{aligned}
& P_{m}^{U P F C}=r B_{s} V_{m}^{2} \sin \gamma-r B_{s} V_{m} V_{n} \sin \left(\delta_{m n}+\gamma\right) \\
& Q_{m}^{U P F C}=r B_{s} V_{m}^{2} \cos \gamma
\end{aligned}
$$




$$
\begin{aligned}
& P_{n}^{U P F C}=-r B_{s} V_{m} V_{n} \sin \left(\delta_{m n}+\gamma\right) \\
& Q_{n}^{U P F C}=r B_{s} V_{m} V_{n} \cos \left(\delta_{m n}+\gamma\right)
\end{aligned}
$$

\section{B. Current Injection modeling of UPFC}

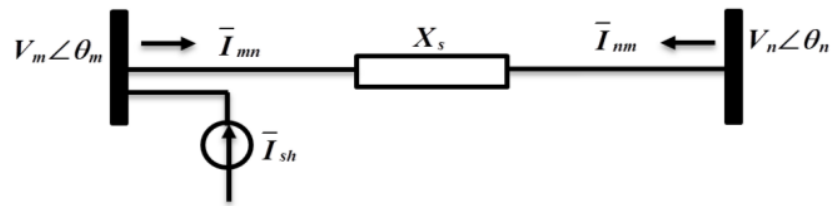

Fig. 2. CI model of UPFC

The current injection model of UPFC is shown in Fig. 2 referred from [14]. Final elements of equivalent power injections are:

$$
\begin{aligned}
& P_{m}^{U P F C}=-B_{s} V_{m} V_{n} \sin \theta_{m n}-r B_{s} V_{m} V_{n} \sin \left(\theta_{m n}+\gamma\right) \\
& Q_{m}^{U P F C}=-B_{s} r V_{m}^{2} \cos \gamma-B_{s} V_{m} V_{n} \cos \theta_{m n}-V_{m}^{2} B_{s} \\
& P_{n}^{U P F C}=B_{s} V_{m} V_{n} \sin \theta_{m n}+r B_{s} V_{m} V_{n} \sin \left(\theta_{m n}+\gamma\right) \\
& Q_{n}^{U P F C}=-B_{s} r V_{m} V_{n} \cos \left(\theta_{m n}+\gamma\right)-B_{s} V_{m} V_{n} \cos \theta_{m n}+V_{n}^{2} B_{s}
\end{aligned}
$$

\section{PROBLEM FORMULATION}

The objective functions can be expressed

$$
\begin{array}{ll}
\text { Min } F_{l}(x, y) & \\
\text { Subject to: } & g(x, y)=0, \\
& h(x, y) \leq 0
\end{array}
$$$$
\forall l=1,2, \ldots \ldots . N
$$

\section{A. Objective Functions}

i). Minimization of total real power loss It can be expressed as

$$
F_{1}=\min \left(T P_{\text {LOSS }}\right)=\sum_{m=1}^{N_{\text {line }}} P_{L O S S, m} M W
$$

ii). Minimization of Generation Cost It can be expressed as

$$
F_{2}=\min \left(F_{m}\left(P_{G_{m}}\right)\right)=\sum_{m=1}^{N_{G}} a_{m} P_{G_{m}}^{2}+b_{m} P_{G_{m}}+c_{m} \$ / h r
$$

\section{B. Constraints}

i) Equality Constraints:

$\sum_{m=1}^{N_{G}} P_{G_{m}}-P_{D}-P_{L}=0 ; \sum_{m=1}^{N_{G}} Q_{G_{m}}-Q_{D}-Q_{L}=0 ;$

where, $P_{D}, Q_{D}$ are the total active and reactive power demands.

ii) In equality Constraints

- Generator

$$
V_{G_{m}}^{\min } \leq V_{G_{m}} \leq V_{G_{m}}^{\max }
$$

$$
Q_{G_{m}}^{\min } \leq Q_{G_{m}} \leq Q_{G_{m}}^{\max }, \quad \forall m \in N_{G}
$$

- Voltage and transformer tap settings

$$
\begin{aligned}
& V_{m}^{\min } \leq V_{m} \leq V_{m}^{\max } \\
& T_{m}^{\min } \leq T_{m} \leq T_{m}^{\max } \\
& \mathrm{m}=1,2, \ldots N_{T}
\end{aligned}
$$$$
\mathrm{m}=1,2, \ldots \quad N_{B}
$$

- Power generation limits

$$
P_{G_{m}}^{\min } \leq P_{G_{m}} \leq P_{G_{m}}^{\max }, \quad \forall m \in N_{G}
$$

- Reactive power

$$
Q_{C_{m}}^{\min } \leq Q_{C_{m}} \leq Q_{C_{m}}^{\max }, \quad \mathrm{m}=1,2, \ldots N_{C}
$$

- Line loadings

$$
S_{l_{m}} \leq S_{l_{m}^{\max }}^{l}, \quad \mathrm{~m}=1,2, \ldots N_{\text {line }}
$$

iii).UPFC constraints

$$
0 \leq r \leq 0.1 ; 0 \leq \gamma \leq 360^{\circ} ; 0 \leq X_{s} \leq 0.1
$$

\section{SQUIRREL SEARCH ALGORITHM}

SSA is based on the dynamic foraging technique and gliding locomotive mechanism of flying squirrels. Basically squirrel used to have two types of nuts; one is hickory nuts which are rarely available and can be stored for longer duration and second is acron nuts which are available in abundance but can be consumed at same instance.

Squirrels are considered to active during summer so they rigorously searches for the food, and after finding acron nuts they consume them immediately and fulfill their daily needs then they start searching for hickory nuts so that they can store them for winters and can increase their lifespan. Squirrel with hickory nut is shown in Fig. 3.

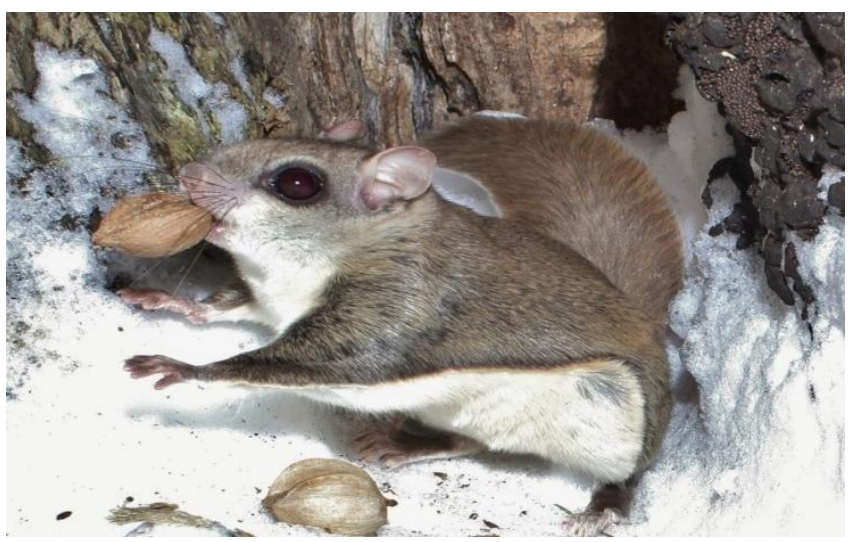

Fig. 3. Squirrel with hickory nut

Here in this algorithm, we considered s number of squirrels locating at $\mathrm{s}$ number of trees and among which only one is hickory nut tree, three is acron tree and remaining are the general trees. So squirrels on general tree tries to reach at acron trees or at hickory nut tree and squirrels on acron trees tries to move towards the hickory nut tree.

\section{A. Steps to implement Squirrel Search Algorithm (SSA)}

1. Initialize the parameters of SSA i.e., number of squirrels, dimension and maximum number of iterations. 
2. Initially generates the random location for the $\boldsymbol{s}$ squirrels by following equation:

$$
S_{i}=S_{l}+U(0,1) \times\left(S_{u}-S_{l}\right)
$$

where, $S_{l}$ and $S_{u}$ are lower and upper bounds respectively of $i^{\text {th }}$ squirrel in $\mathrm{j}^{\text {th }}$ dimension and $\mathrm{U}(0,1)$ random number in the range of $[0,1]$.

3. Calculate the fitness for each location of flying squirrels and then sort them in accordance to its fitness values.

4. Fittest value will be of that flying squirrel, located at hickory nut tree, then next three fit values will be of flying squirrel which is on acron trees and then remaining will be on normal trees.

5. Select any of the flying squirrel located at normal trees and target them to directs toward hickory nut tree and remaining to the acron trees.

\section{while (threshold value not achieved)}

\section{6. for $i=1$ to 1 1(n1=number of squirrels present on acorn} trees and directed towards hickory nut tree)

if $R 1 \geq 0.1$

else

$S_{a t}^{t+1}=$ Random position will be allocated

end

7. for $i=1$ to $n 2$

if $R 2 \geq 0.1$

$S_{n t}^{t+1}=S_{n t}^{t}+R_{2} \times\left(S_{a t}^{t}-S_{n t}^{t}\right)$

else

$S_{n t}^{t+1}=$ Random position will be allocated

end

8. for $i=1$ to $n 3$

if $R 3 \geq 0.1$

$S_{n t}^{t+1}=S_{n t}^{t}+R_{3} \times\left(S_{h t}^{t}-S_{n t}^{t}\right)$

else

$S_{n t}^{t+1}=$ Random position will be allocated

end

9. Calculate the seasonal constant by using following equation:

$$
\left.S_{c}^{t}=\sqrt{\sum_{k=1}^{d}\left(S_{a t, k}^{t}\right.}-S_{h t, k}^{t}\right)^{2}
$$

10. Verify the seasonal monitoring condition i.e. $S_{c}^{t}<S_{\min }$, if it found valid then find levy flight operator using equation:

$$
\operatorname{Levy}(x)=0.01 \times \frac{r_{1}}{\left|r_{2}\right|^{1 / \alpha}} \times \gamma,
$$

Where, $r_{1}$ and $r_{2}$ are random numbers in the range of $(0,1)$ and

$$
\gamma=\left(\frac{\Gamma(1+\alpha) \times \sin (0.5 \pi \alpha)}{\Gamma\left(\frac{1+\alpha}{2}\right) \times \alpha \times 2^{0.5(\alpha-1)}}\right)^{\frac{1}{\alpha}},
$$

where, $\alpha$ is a constant having value equal to 1.5 . Then, randomly relocate the flying squirrel by following equation:

$$
S_{n t}^{n e w}=S_{l}+\operatorname{Levy}(n) \times\left(S_{u}-S_{l}\right)
$$

11. Minimum value of seasonal constant, $S_{\min }$ can be computed as:

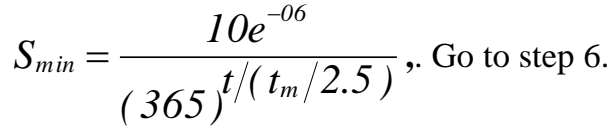

end

12. The squirrel present on hickory nut tree will be considered as optimal value of objective.

end

\section{RESULTS AND ANALYSIS}

The proposed SSA has been verified on IEEE-30 bus system by solving the OPF problems. The details are given in[15].

\section{A. Single Objective Optimization}

Hereby, single objectives functions are considered for solving OPRD. Initially, the generation fuel cost is being considered and solved to obtain an optimal value, thus it validates the proposed SSA method and then the results are being compared with other existing algorithms. The optimal values of control variables for generation fuel cost are shown in Table 1 and we can say that we can say that the generation fuel cost is minimized to a better value by implementation of SSA in comparison to other existing algorithms. In Fig. 4, convergence characteristics is shown in comparison with other existing algorithms and it is clearly analyzed that the proposed method initiates with better function value and converges in lesser number of iterations.

Table- I: Solution for generation fuel cost minimization

\begin{tabular}{|l|c|c|c|}
\hline Variables & PSO [16] & HSCA [16] & SSA \\
\hline PG1, MW & 178.556 & 176.87 & 173.4146 \\
\hline PG2, MW & 48.6032 & 49.8862 & 48.78894 \\
\hline PG5, MW & 21.6697 & 21.6135 & 20.9484 \\
\hline PG8, MW & 20.7414 & 20.8796 & 24.0219 \\
\hline PG11, MW & 11.7702 & 11.6168 & 12.92311 \\
\hline PG13, MW & 12 & 12 & 12.37984 \\
\hline V1, p.u. & 1.1 & 1.057 & 1.1 \\
\hline V2, p.u. & 0.9 & 1.0456 & 0.983134 \\
\hline V5, p.u. & 0.9642 & 1.0184 & 1.028617 \\
\hline V8, p.u. & 0.9887 & 1.0265 & 1.032742 \\
\hline V11, p.u. & 0.9403 & 1.057 & 0.912917 \\
\hline V13, p.u. & 0.9284 & 1.057 & 1.074455 \\
\hline T 6-9, p.u. & 0.9848 & 1.0254 & 0.972776 \\
\hline T 6-10, p.u. & 1.0299 & 0.9726 & 1.024313 \\
\hline T 4-12, p.u. & 0.9794 & 1.006 & 1.072996 \\
\hline Tap 28-27, p.u. & 1.0406 & 0.9644 & 1.028331 \\
\hline Qc 10, p.u. & 9.0931 & 25.3591 & 19.7065 \\
\hline Qc 24, p.u. & 21.665 & 10.6424 & 7.346241 \\
\hline $\begin{array}{l}\text { Generation fuel } \\
\text { cost \$/h }\end{array}$ & $\mathbf{8 0 3 . 4 5 4}$ & $\mathbf{8 0 2 . 0 3 4}$ & $\mathbf{8 0 1 . 8 1 3 4}$ \\
\hline
\end{tabular}


Furthermore, the generation fuel cost and transmission line losses are considerd with PIM and CIM of UPFC. Significantly, UPFC is placed between buses 10-17. The results obtained for both the objectives in Table 2 . It can be observed that without any FACTS device generation fuel cost obtained is $801.8134 \$ / \mathrm{h}$ which is further minimized by using UPFC but it can also be seen that generation fuel cost

Table- II: Single objective OPF results of generation fuel cost and transmission line losses with PIM and CIM of UPFC

\begin{tabular}{|c|c|c|c|c|c|c|}
\hline \multirow[b]{3}{*}{ Variables } & \multirow{2}{*}{\multicolumn{3}{|c|}{ Generation fuel cost, $\$ / \mathbf{h}$}} & \multirow{2}{*}{\multicolumn{3}{|c|}{ Transmission line losses, MW }} \\
\hline & & & & & & \\
\hline & $\begin{array}{c}\begin{array}{c}\text { Without FACTS } \\
\text { devices }\end{array} \\
\end{array}$ & PIM of UPFC & CIM of UPFC & $\begin{array}{c}\text { Without FACTS } \\
\text { devices }\end{array}$ & PIM of UPFC & CIM of UPFC \\
\hline PG1, MW & 173.4146 & 177.817 & 177.7548 & 64.926 & 60.81635 & 51.50466 \\
\hline PG2, MW & 48.78894 & 49.6322 & 48.82273 & 67.781 & 80 & 80 \\
\hline PG5, MW & 20.9484 & 20.7973 & 21.488 & 50 & 50 & 50 \\
\hline PG8, MW & 24.0219 & 19.8118 & 21.01923 & 35 & 33.50448 & 35 \\
\hline PG11, MW & 12.92311 & 12.2234 & 11.21453 & 30 & 30 & 30 \\
\hline PG13, MW & 12.37984 & 12.2959 & 12 & 40 & 32.77332 & 40 \\
\hline V1, p.u. & 1.1 & 1.1 & 1.1 & 1.0998 & 1.082045 & 1.1 \\
\hline V2, p.u. & 0.983134 & 1.0868 & 1.043946 & 1.02653 & 1.026591 & 1.03651 \\
\hline V5, p.u. & 1.028617 & 1.0658 & 1.06612 & 1.06537 & 1.055954 & 1.081808 \\
\hline V8, p.u. & 1.032742 & 1.07204 & 1.071553 & 1.07114 & 1.056863 & 1.077256 \\
\hline V11, p.u. & 0.912917 & 1.08935 & 1.1 & 1.0289 & 0.968815 & 1.01721 \\
\hline V13, p.u. & 1.074455 & 0.99026 & 1.1 & 1.02694 & 1.026339 & 1.099327 \\
\hline T 6-9, p.u. & 0.972776 & 0.93913 & 1.00552 & 1.1 & 1.004835 & 0.964174 \\
\hline T 6-10, p.u. & 1.024313 & 1.078 & 1.005329 & 1.08777 & 0.972508 & 0.989744 \\
\hline T 4-12, p.u. & 1.072996 & 0.94419 & 1.025489 & 1.1 & 0.999987 & 0.941364 \\
\hline T 28-27, p.u. & 1.028331 & 0.97005 & 0.982578 & 1.0721 & 0.945153 & 0.962597 \\
\hline Qc 10 , p.u. & 19.7065 & 8.03898 & 12.92405 & 25 & 6.031126 & 23.32114 \\
\hline Qc 24, p.u. & 7.346241 & 28.0015 & 12.00949 & 21.985 & 16.02577 & 10.38963 \\
\hline $\mathrm{r}$ & NA & 0.08629 & 0.034822 & NA & 0.0344954 & 0.02387 \\
\hline$\gamma$ & NA & 96.5632 & 109.541 & NA & 170.5344 & 181.3545 \\
\hline & NA & 0.04563 & 0.00546 & NA & 0.08932 & 0.04212 \\
\hline Generation fuel cost \$/h & 801.8134 & 800.736 & 799.8826 & 899.664 & 895.3662 & 890.455 \\
\hline
\end{tabular}

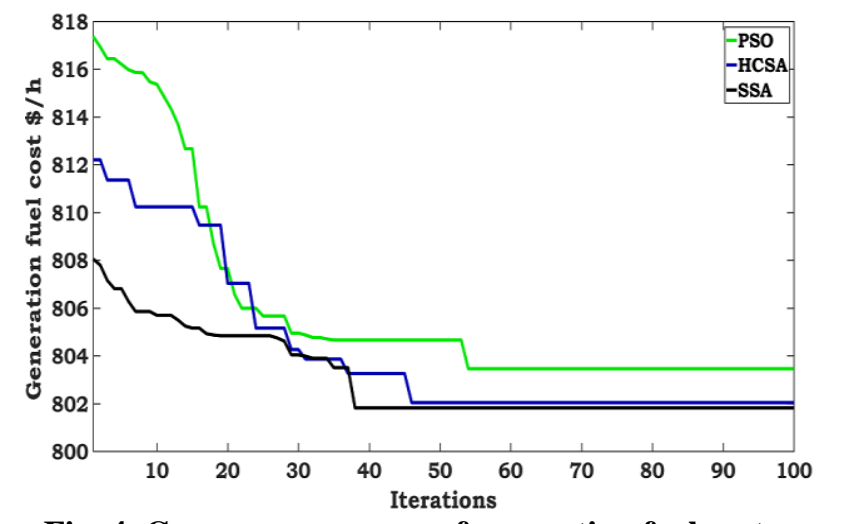

Fig. 4. Convergence curve of generation fuel cost

Tig. obtained with CIM of UPFC is $799.8826 \$ / h$ while with PIM of UPFC is $800.736 \$ / h$, thus we can say that CIM is best technique for modeling UPFC. Similarly, transmission line losses obtained with CIM of UPFC is 3.103MW which is better value obtained in comparison to the PIM of GIPFC.

\section{CONCLUSION}

In this research work, a novel squirrel search algorithm (SSA) was effectively used to solve the OPRD problem for IEEE-30 bus system satisfying equality and in-equality constraints. Cost of generation and transmission line losses are minimized more optimally as compared to other existing algorithms. The result analyzed proves that the proposed method yields to good convergence curve in comparison to other existing methods. Furthermore, incorporation of UPFC in power system results the better solution for the objectives in comparison with the system with FACTS controller. It is also being observed that among both the considered modeling technique, 
CIM of UPFC is more reliable as compared to PIM of UPFC. Hence, we can say that solution of OPRD using SSA with incorporation of CIM of UPFC is a better alternative and more feasible method.

\section{REFERENCES}

1. Enrique Acha et al., "FACTS: Modeling and Simulation in Power Networks", Wiley publication, 2004.

2. JavadLavei, AndersRantzer, Stephen Low, "Power flow optimization using positive quadratic programming", (2011) 18th IFAC, Italy

3. El-Hawary, J.A.M.M.E., Adapa, R, “A review of selected optimal power flow literature to 1993. part i: Nonlinear and quadratic programming approaches", IEEE transactions on power systems, Vol. 14, (1999A), No. 1.

4. O. Alsac, B. Stott, "Optimal load flow with steady-state security, IEEE Transactions on Power Systems", (1973), 484-3.

5. David I. Sun, Bruce Ashley, Brian Brewer, A. Hughes, William Tinney, "Optimal power flow by newton approach", IEEE Transaction PAS, Volume - 103, (1984), No.10.

6. J.T.Ma, L.L.Lai, "Application of genetic algorithm to optimal reactive power dispatch including voltage-dependent load models", IEEE-ICEC, 1995, PP:5-10.

7. S. Duman, Y. Sönmez, U. Güvenç, N. Yörükeren, "Optimal reactive power dispatch using a gravitational search algorithm", IET Gener. Transm. Distrib. 6, (2012), 563.

8. W.S. Jwo, "Hybrid expert system and simulated annealing approach to optimal reactive power planning", IEE Proceeding GTD 142, (1995), 38.

9. A.A. El-Fergany, H.M. Hasanien, "Single and multi-objective optimal power flow using grey wolf optimizer and differential evolution algorithms", Electrical Power Component System 43 (2015) 1548-1559.

10. M. Abido, "Optimal power flow using particle swarm optimization", International Journal Electr. Power Energy Syst. 24, 2002, PP:563-571.

11. S.S. Jadon, J.C. Bansal, R. Tiwari, H. Sharma, "Artificial bee colony algorithm with global and local neighborhoods", IJSA, 8 (2014), 1-13.

12. MohitJain, VijanderSingh, Asha Rani, "A novel nature-inspired algorithm for optimization: Squirrel search algorithm", swarm and evolutionary computation, 44, 2019, PP:148-175.

13. MithuSarkar, "Load Flow Studies with UPFC Power Injection Model", thesis M.Tech in Power Control and Drives, NIT Rorkela, 2013.

14. M. Balasubba Reddy, Divyanshi Dwivedi, "Incorporation of Current Injection Modelling of UPFC and Analyzing Power Flow Solution using Criss Cross Optimization Algorithm", Proceedings of 2019 3rd IEEE International Conference on Electrical, Computer and Communication Technologies, Vol. 1, (2019).

15. M. A. Abido, "Optimal Power Flow Using Tabu Search Algorithm", Electric Power Components and Systems Volume 30, 2002, Issue 5.

16. M. Balasubba Reddy, "A solution to the Multi Objective Optimization problems with FACTS devices using NSHCSA including practical constraints", IEEE-ICPCSIE, (2017).

\section{AUTHORS PROFILE}

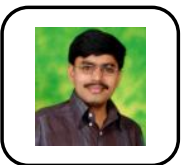

Dr. M Balasubbareddy, research focuses on Optimization Techniques, IOT, smart grid, design of Photovoltaic systems and electrical distribution analysis. He uses MATLAB programming and optimization techniques including Hardware experience design of PV systems with SCADA control. Three projects were executed with off grid and on grid systems with support of Solar Energy Corporation of India (SECI). In collaboration with a Chennai based company Industrial Controls \& Drives (India) Pvt. Ltd.(ICD), his team has developed an energy management system for integrating grid and solar power generation capacity of $400 \mathrm{kWp}$ at SV Colleges, Tirupati (Previous working College). His research has developed multi-objective hybrid optimization algorithm in the presence of FACTS devices. He published significant number of referred journals in the area of Multi-objective optimization techniques and design of solar energy systems.

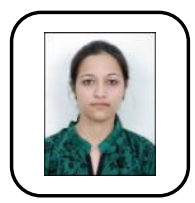

Divyanshi Dwivedi, received the B.tech degree from Rajasthan Technical University in 2014, M.E. degree in Power System and Power Electronics from CBIT, Hyderabad in 2019. Her research interests include power system, FACTS Controller and Optimization techniques. 